\title{
Bayesian reconstruction of images of objects with high-density inclusions with suppression of artifacts
}

\author{
Sergei Zolotarev ${ }^{1}$, Valery Vengrinovich ${ }^{1}$, Mohsen Mirzavand ${ }^{2}$, Mieteeg Mukhtar ${ }^{2}$ and \\ Ivan Georgiev 3,4,* \\ ${ }^{1}$ National Academy of Sciences of Belarus, Institute of Applied Physics, Minsk, Belarus \\ ${ }^{2}$ Belarusian National Technical University, Minsk, Belarus \\ ${ }^{3}$ Institute of Information and Communications Technologies, Bulgarian Academy of Sciences, \\ Sofia, Bulgaria \\ ${ }^{4}$ Instituteof Mathematics and Informatics, Bulgarian Academy of Sciences, Sofia, Bulgaria
}

\begin{abstract}
The technology of three-dimensional Bayesian tomographic reconstruction of homogeneous objects with high-density inclusions is developed. The approach is based on preliminary correction of projections by extracting the data corresponding to $\mathrm{X}$-rays passing through a highdensity region, and replacing it with synthesized data obtained by twodimensional interpolation. An original method for selecting interpolation points is proposed and a mathematical algorithm is described that ensures the implementation of two-dimensional interpolation correction of projections.
\end{abstract}

\section{Introduction}

There is a wide class of problems of X-ray tomography, solutions of which are piecewiseconstant functions and, accordingly, of industrial objects leading to the formulation of such tasks. Such control objects include, for example, so-called piecewise-homogeneous structures, objects or systems, which have different homogeneous parts with constant coefficients of linear attenuation. The boundaries between the heterogeneous parts of complex industrial products are usually sharp, without a smooth transition from one constructive part to another. Such heterogeneous media with a piecewise homogeneous structure can be adequately described by precisely defining the boundary surfaces that separate the homogeneous parts of such media.

We will consider control objects that have local phase inclusions inside, and we will assume that within each phase, the coefficient of linear attenuation of X-ray radiation is fairly homogeneous, and its numerical value differs significantly from the coefficient of linear attenuation of the main material, which, in general, can be heterophase structure. The number of local phase inclusions inside the main material of the object will be assumed to be arbitrary, but, of course, finite. We will also assume that the values of the attenuation

\footnotetext{
* Corresponding author: ivan.georgiev@parallel.bas.bg
} 
coefficients of the desired phase inclusions may differ from each other, but they are all different from the coefficients of linear attenuation of the limiting material that limits them. At tomographic reconstruction of such industrial objects, for example, plastic products, inside which various small metal inserts are pressed, there are strong artifacts that create strong interference to the study of the internal structure and geometric configuration of the components of the product.

The main reasons for these artifacts are: 1) the effect of Beam Hardening of X-rays; 2) $\mathrm{X}$-Ray scattering; and 3) a high level of noise component in the projection data and others. One of the known approaches is the method of preliminary correction of projections by extracting data corresponding to X-rays passing through a metal implant and replacing them with synthesized data obtained by linear or polynomial interpolation [1-4]. Next, we will briefly describe the technology of identifying homogeneous objects proposed by us, for example, the control of products made on $3 \mathrm{D}$ printers and considered a simple, but an effective enough way to overcome the above difficulties, which turned out to be useful for solving other problems.

Moreover, an original method of interpolation points selection will be offered and a mathematical algorithm providing implementation of interpolation projection correction will be described in steps.

\section{Theory}

\subsection{The heuristic method of statistical iterative reconstruction}

We develop statistical iterative algorithms of reconstruction using minimize total variations and OpenGL. Iterative methods for tomography based on solving a system of linear algebraic equations

$$
A \boldsymbol{x}=\boldsymbol{p}
$$

We are experienced in this work a heuristic iterative algorithm on theoretical grounds close to the algorithm published in [5] but differs primarily by the fact that we use the correction of the current approach by minimizing the residuals for the radial integrals, not intensities. The proposed reconstruction algorithm (Heuristic Bayesian Inference Engine, HBIR) [6] can be written as

$$
x_{j}^{(k+1)}=x_{j}^{(k)}\left[1+\lambda^{(k)} \sum_{i=1}^{I} \frac{A_{i j}}{\sum_{j=1}^{J} A_{i j}} \frac{\left(p_{i}-\sum_{j=1}^{J} A_{i j} x_{j}^{(k)}\right)}{\sum_{j=1}^{J} A_{i j} x_{j}^{(k)}}\right],
$$

where $x_{j}^{(k)}$ - the value of the $j$-th component of the vector of unknowns at the $k$-th iteration, $A_{i j}$ - elements of the projection matrix, $p_{i}$ - the value of the measured radial integral in the pixel number $i, \lambda^{(k)}$ - sequence relaxation parameter values $\left(0<\lambda^{(k)}<1\right)$.

Correction algorithm represented by the formula (2) is nearly as convenient for parallelization using OpenGL graphics library, as well as the famous SART algorithm [7]. Direct projection of a three-dimensional texture, containing an image of the current approach is the same as for the SART algorithm, which is described in detail in [8]. The 
difference lies in the fact that its implementation must use two additional two-dimensional textures. In one of them contains a correction image. The second texture contains an image of layer of the corrected three-dimensional texture. Correcting the image is first projected perspective [9] to the place where is the corrected layer, and then multiplied with the corrected image of layer and recorded in its place. For multiplication of two-dimensional texture, use texture mapping to the object with GL_MODULATE regime.

\subsection{The principle of extraction from projections of local regions with images of inhomogeneities}

This method assumes that for each of the X-ray projections it is possible to segment local regions with images of inhomogeneities. Thus, the question arises: how, having projective views containing the projections of voxels with maximum density, it is correct to extract them from the original projection species containing the values of the ray integrals.

We describe the simple algorithm we proposed and tested in practice, which provides the solution to this problem. Consider the following Figure 1, which shows the projection, with the local area on it being the image of a high-density heterogeneity. Through a point inside the local region of inhomogeneity, a set of straight lines located through equal angular intervals is conducted.

The interpolation points necessary to obtain a new approximated value in the pixel of the digital image, are located at the ends of segments emerging from the point under consideration and terminating outside the boundary of the local inhomogeneity.

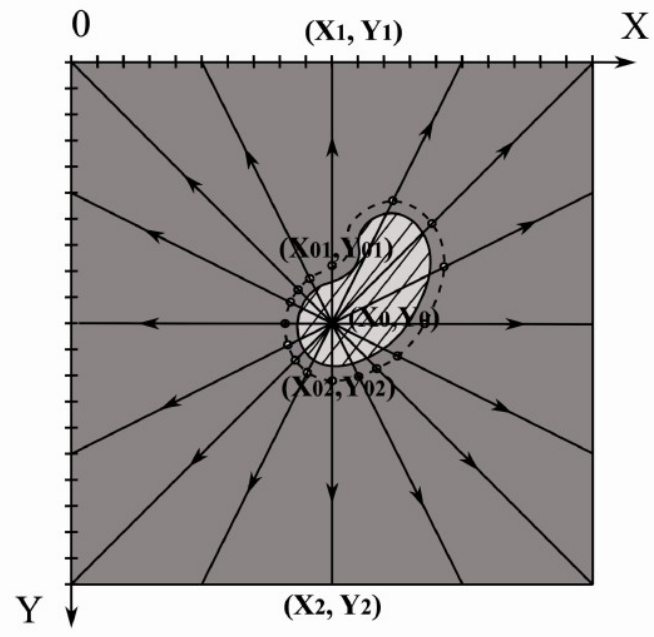

Fig 1. Projections with the local area of high-density heterogeneity

\section{Interpolation algorithm for extractable heterogeneity values}

Input: The number of two-dimensional projections $-\boldsymbol{n} \_\boldsymbol{p r j}$, Projections with Beam Integrals $\boldsymbol{P}^{(l)}, l=1, \ldots, n \_p r j$ with dimensions along the axes $\underline{O X}$ and $\underline{O Y}-\mathbf{s i z e} \boldsymbol{x}$, size_y, defined as one-dimensional vectors $\boldsymbol{P}^{(l)}=\left(P^{(l)}{ }_{1}, \ldots, P^{(l)}{ }_{n}\right.$ pix $)$, where $\boldsymbol{n} \_\boldsymbol{p i x}=$ size_x $\boldsymbol{x}^{*}$ size $\_\boldsymbol{y}$ and

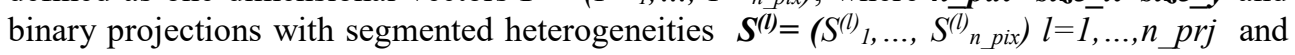


the same dimensions size_x и size_y, $\boldsymbol{n} \_$lin - the number of straight lines that will be used to estimate the interpolated value.

Output: Adjusted projections $\boldsymbol{P}^{(l)}, l=1, \ldots, n \_p r j$ with extracted pixel values belonging to high-density heterogeneities.

1: We organize cycle $\mathbf{1}$ in the number of projections $\mathbf{n} \_$prj: $\boldsymbol{f o r}\left(l=1 ; l<=n \_\right.$prj; $\left.l++\right)\{\boldsymbol{B o d y}$ of cycle 1 \}

2: Inside the body of cycle 1 simultaneously for a projection with ray integrals $\boldsymbol{P}^{(\boldsymbol{l}) \text {, }}$ and the corresponding segmented (binary) projection $\boldsymbol{S}^{(l)}$ organize cycle $\mathbf{2}$ in terms of the number of pixels $n \_$pix: for $\left(n=1 ; n<=n \_\right.$pix; $\left.n++\right)\{$ Body of cycle 2$\}$

3: Inside the body of cycle 2 for each pixel of the segmented projection $S^{(l)}{ }_{n}$ we check that condition

4: if The pixel value of the segmented projection $S^{(l)}{ }_{n}$ is nonzero then

5: From the value of the index n, we determine the discrete coordinates $(i 0, j 0)$ this pixel koord_pixel(n,size_x, \&i0,\&j0), and on them we further determine its Cartesian coordinates $\left(X_{0}, Y_{0}\right)$.

6: We carry out the projections with ray integrals $\boldsymbol{P}^{(l)}$ through the point $\left(X_{0}, Y_{0}\right) n_{-}$lin direct lines up to the intersection with its boundaries at points $\left(X^{(k)}{ }_{1}, Y^{(k)}{ }_{1}\right)$ и $\left(X^{(k)}{ }_{2}, Y^{(\bar{k})}{ }_{2}\right)$, where $k \in\left\{1, \ldots, n \_l i n\right\}$. In figure 1 , the k-index for the first line is not shown. These straight lines are located at the same angular intervals relative to each other.

7: Inside the body of cycle 2 , we organize cycle 3 in the number of lines $\boldsymbol{n}$ lin:

for $\left(k=1 ; k<=n \_l i n ; k++\right)\{$ Body of cycle 3$\}$

8: Inside the body of cycle 3 for each straight line, determine the number of pixels $m_{-} 1$, lying between the points $\left(X_{0}, Y_{0}\right)$ и $\left(X^{(k)}{ }_{1}, Y^{(k)}{ }_{l}\right)$, their discrete coordinates $i_{-} 1_{m}, j_{-} 1_{m}$, где $m=1, \ldots, m_{-} 1$, indices $n_{-} 1_{1}, \ldots, n_{-} 1_{m_{-} l}$, where $n_{-} 1_{m} \in\left\{1, \ldots, n_{-}\right.$pix $\}$and have them in the order from the point $\left(\bar{X}_{0}, Y_{0}\right)$ to the point $\left(X^{(k)}{ }_{1}, Y^{(k)}{ }_{1}\right)$. Similarly, for each straight line, determine the number of pixels $m_{-} 2$, lying between points $\left(X_{0}, Y_{0}\right)$ и $\left(X^{(k)}{ }_{2}, Y^{(k)}{ }_{2}\right)$, their discrete coordinates and have them in the order from the point $\left(X_{0}, Y_{0}\right)$ к точке $\left(X^{(k)}{ }_{2}, Y^{(k)}{ }_{2}\right)$ and so on.

9: Inside the body of cycle 3 , arrange loop 4 on the number of pixel points $\boldsymbol{m}_{-} \mathbf{1}$ : for $\left(m=1 ; m<=m_{-} 1 ; m++\right)\{$ Body of cycle 4$\}$

10: Inside the body of cycle 4, we move along the pixels from the point $\left(X_{0}, Y_{0}\right)$ to the point $\left(X^{(k)}{ }_{1}, Y^{(k)}{ }_{1}\right)$ and for each pixel of the segmented projection $S^{(l)}{ }_{n}$ with the current index $n_{-} 1_{m}$ further we verify that condition

11: if The pixel value of the segmented projection $S^{(l)}{ }_{n}$ with the current index $n_{-} 1_{m}$ is nonzero then

12: We keep the current values of the indices, discrete and Cartesian coordinates, which are determined by discrete coordinates, as well as the number of pixels passed

$$
n_{-} l^{(k)}=n_{-} 1_{m} ; i_{-} 1^{(k)}=i_{-} 1_{m} ; j_{-} 1^{(k)}=j_{-} 1_{m} ; X_{-} 1^{(k)}=X_{-} 1_{m} ; Y_{-} 1^{(k)}=Y_{-} 1_{m} ; m_{-} l^{(\mathrm{k})}=m ;
$$

13: else We leave the body of cycle 4

\section{4: end if}

15: Inside the body of cycle 3 , we organize cycle 5 in terms of the number of pixel points m_2 : for $\left(m=1 ; m<=m \_2 ; m++\right)\{$ body of cycle 5\}

16: Inside the body of cycle 5, we move along the pixels from the point $\left(X_{0}, Y_{0}\right)$ to the point $\left(X^{(k)}{ }_{2}, Y^{(k)}{ }_{2}\right)$ and for each pixel of the segmented projection $S^{(l)}{ }_{n}$ with the current index $n_{-} 2_{m}$ we check that condition

17: if The pixel value of the segmented projection $S^{(l)}{ }_{n}$ with the current index $n_{-} 2_{m}$ is nonzero then

18: Save the current values of the indexes, discrete and Cartesian coordinates, which are determined by discrete coordinates, as well as the number of traversed pixels

$$
n \_2^{(k)}=n \_2 m ; i \_2^{(k)}=i \_2 m ; j \_2^{(k)}=j \_2 m ; X \_2^{(k)}=X \_2 m ; Y \_2^{(k)}=Y \_2 m ; m \_2^{(\mathrm{k})}=m ;
$$

19: else We leave the body of cycle 5 


\section{0: end if}

21: Shift by shifting pixels along the current line from the end point $\left(X_{-} l^{(k)}, Y_{-} l^{(k)}\right)$ to the point $\left(X^{(k)}{ }_{1}, Y^{(k)}{ }_{1}\right)$ with the aim that the new point lies outside the region of local inhomogeneity and, thus, redefine the end point:

$$
\begin{aligned}
& X_{-} l^{(k)}=X_{0}+\left(m_{-} l^{(\mathrm{k})}+\text { shift }\right)^{*} \text { parameter } l^{*}\left(X^{(k)}{ }_{1}-X_{0}\right) ; \\
& Y_{-} l^{(k)}=Y_{0}+\left(m_{-} l^{(\mathrm{k})}+\text { shift }\right) * \text { parameter } l^{*}\left(Y^{(k)}{ }_{1}-Y_{0}\right) ;
\end{aligned}
$$

In accordance with the displacement of the end point, we also redefine the values of the indices and discrete coordinates for the end point $\left(X_{-} l^{(k)}, Y_{-} l^{(k)}\right)$. Shift by shifting pixels along the current line from the end point $\left(X_{-} 2^{(k)}, Y_{-} 2^{(k)}\right)$ to the point $\left(X^{(k)}{ }_{2}, Y^{(k)}{ }_{2}\right)$ with the aim that the new point lies outside the region of local inhomogeneity and, thus, redefine the end point:

$X \_2^{(k)}=X_{0}+\left(m \_2^{(\mathrm{k})}+\text { shift }\right)^{*}$ parameter $2 *\left(X^{(k)}{ }_{2}-X_{0}\right)$;

$Y_{-} 2^{(k)}=Y_{0}+\left(m_{-} 2^{(\mathrm{k})}+\right.$ shift $) *$ parameter $2 *\left(Y^{(k)}{ }_{2}-Y_{0}\right)$;

In accordance with the displacement of the end point, we also redefine the values of the indices and discrete coordinates for the end point $\left(X \_2^{(k)}, Y \_2^{(k)}\right)$.

22: Determine the distance between the end points and the distance from the end points to the point $\left(X_{0}, Y_{0}\right)$

dl_12 $2^{(k)}=\operatorname{distance}\left(X \_1^{(k)}, Y \_1^{(k)}, X \_2^{(k)}, Y \_2^{(k)}\right)$;

dl_ $l^{(k)}=\operatorname{distance}\left(X_{-} \overline{1}^{(k)}, Y_{-}^{-} \bar{l}^{(k)}, X_{0}, Y_{0}\right)$;

dl_ $2^{(k)}=\operatorname{distance}\left(X_{-} 2^{(k)}, Y_{-}^{-} 2^{(k)}, X_{0}, Y_{0}\right)$;

23: We finish cycle $\overline{3}$ in the number of lines $\boldsymbol{n}_{-}$lin.

24: For the current index $n$, we calculate the interpolated value in pixel

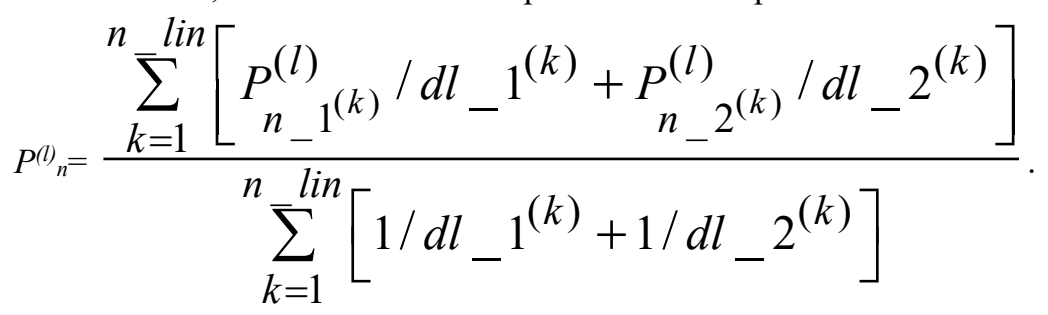

25: else (That is, inside the body of cycle 2, the pixel value of the segmented projection $S$ (1) $n$ is zero) We move on to the next pixel in cycle 2.

\section{6: end if}

27: Finish cycle 2.

28: Finish cycle 1.

\section{Reconstruction image of a two-component object}

The experimental verification of the feasibility of implementing the proposed technology, in particular, suppression of artifacts caused by high-density inclusions, was carried out on Real-Time X-Ray Inspection System of Seifert $x \mid$ cube with $225 \mathrm{kV}$. Figure 2 below shows the projections with ray integrals of the physical phantom of the knee joint, manufactured on a 3D printer for angles 450, 900 and 1350. Figure 3 shows the projections of segmented metal pins. 


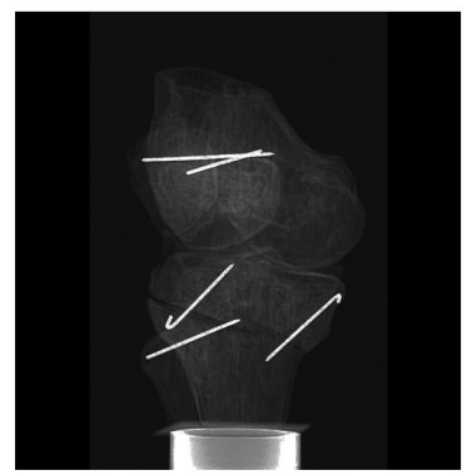

(a)

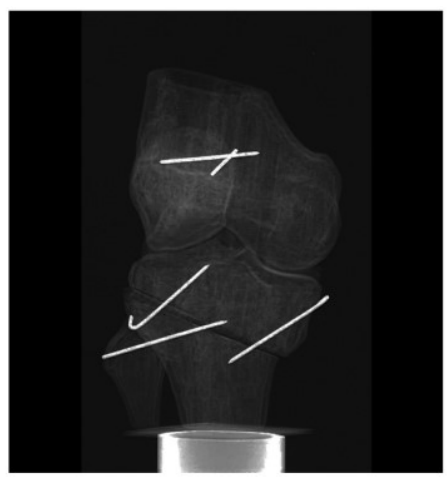

(b)

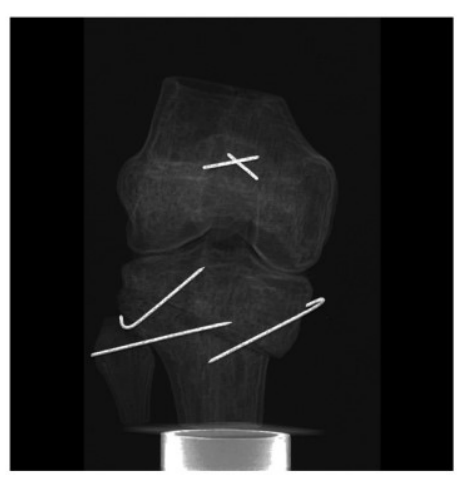

(c)

Fig 2. Projections with ray integrals for angles a) $45^{\circ}$, b) $90^{\circ}$ and c) $135^{\circ}$

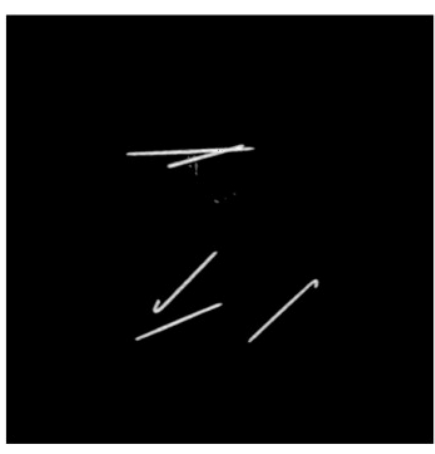

(a)

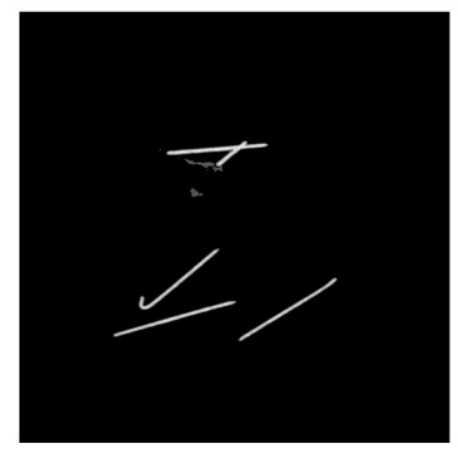

(b)

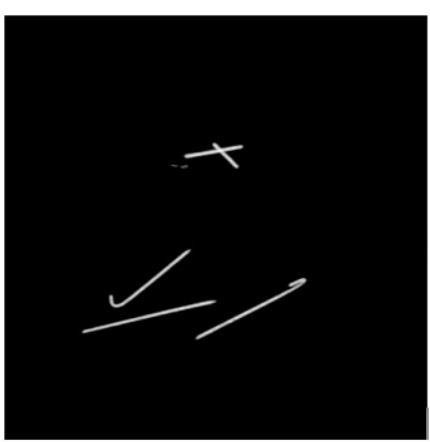

(c)

Fig. 3. Projections of segmented metal pins for angles a) $45^{\circ}$, b) $90^{\circ}$ and c) $135^{0}$

When reconstructing the image of the knee joint, projections with ray integrals produced strong artifacts in the areas of the location of the metal pins that were caused, including, and the effect of "Beam hardening of X-rays." As a result of applying the algorithm described above, extraction of images of high-density materials from joint projections was carried out. Projections of the knee joint with extracted images of nuts for angles 0,90 and 135 are shown in Figure 3.

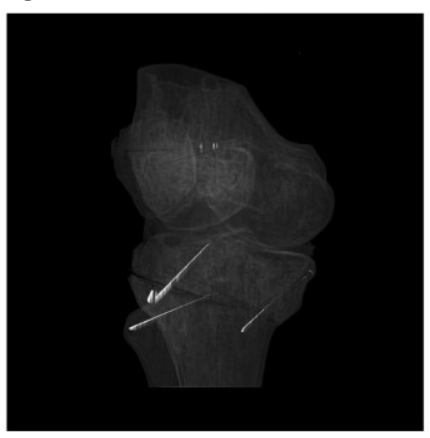

a)

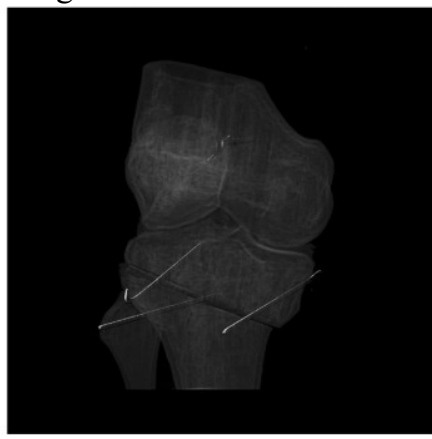

(b)

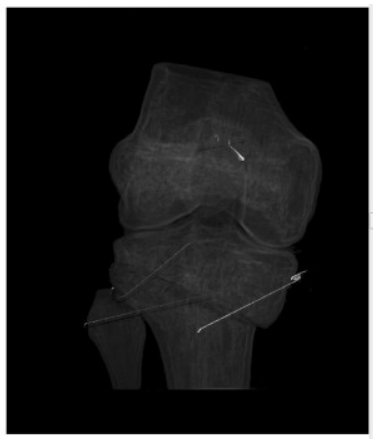

(c)

Fig. 4. Filtered projections with ray integrals for angles a) $45^{\circ}$, b) $90^{\circ}$ and c) $135^{\circ}$ 
As a result of the applied approach, it was possible to carry out a separate reconstruction of the plastic basis of the knee joint. Figure 5 shows a three-dimensional image of the plastic base of the knee joint. Figure 6 shows the cross sections of the $3 \mathrm{D}$ image of the knee joint by the planes XOY and YOZ and also XOZ.

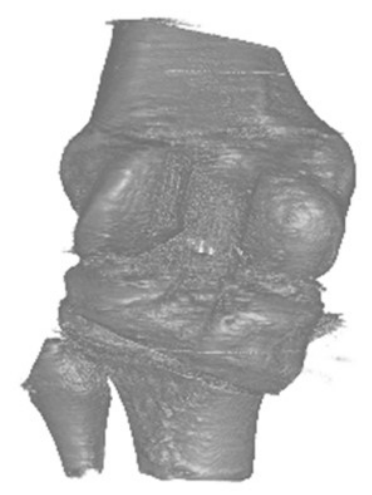

Fig. 5. Three-dimensional image of the plastic base of the knee joint

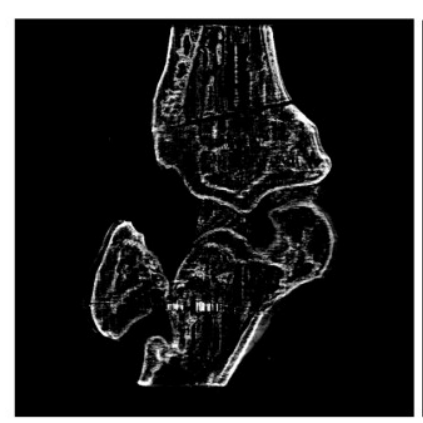

a)

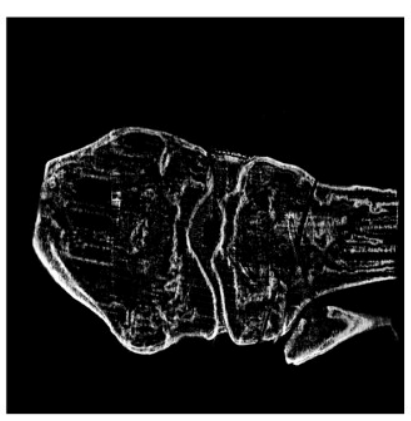

(b)

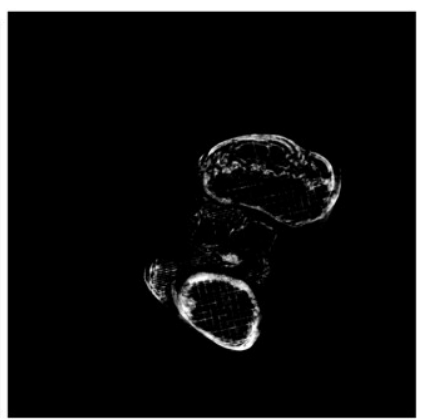

(c)

Fig. 6. Cross sections of the three-dimensional image of the knee joint by the planes: a) XOY and b) $\mathrm{YOZ}$ and also c) $\mathrm{XOZ}$

The proposed method of three-dimensional tomographic reconstruction of objects with high-density inclusions based on preliminary correction of projection images by extracting projection data corresponding to $\mathrm{X}$-rays passing through high-density regions of the reconstruction object showed good efficiency both in tomographic reconstruction of the physical phantom of the knee joint and in the reconstruction of two-component industrial facilities. A new interpolation scheme is proposed for choosing the location of the interpolation points and a mathematical algorithm for its implementation is described in detail.

This work was partially supported by the Joint Research Project "Advanced Tomographic Reconstruction Methods" between IICT-BAS and IAP-NAS of Belarus and Bulgarian NSF Grant DFNI-I02/9. 


\section{References}

1. G.H. Glover, Med. Phys. 8, 6 , 799-807 (1981)

2. W.A. Kalender, Radiology 164, 576-577 (1987)

3. E.Klotz, Medical Imaging IV: PACS System Design and Evaluation, SPIE - The International Society for Optical Engineering 1234, 642-650 (1990)

4. R.M. Lewitt, Optik 50, 3, 189-204 (1978)

5. K. Lange, Image Processing, IEEE Transactions 4, 10, 430 - 1438 (1995)

6. S.A. Zolotarev, V.L. Vengrinovich, M.A. Mirzavand, Conf. Proc. NDT Days 2015, XXIII 2, 165 (2015).

7. A. H.Andersen, J.Ultras.Imaging 6, 1, 81 - 94 (1984)

8. V. Vengrinovich, S Zolotarev, Iterative methods for tomography (Minsk ,Belarusian Science, 2009) (in Russian)

9. M. Segal, SIGGRAPH 92, 26, 249-252, 1992 